\title{
Let-7d inhibits colorectal cancer cell proliferation through the CST1/p65 pathway
}

\author{
JIE JIANG* , HUI-LING LIU* ${ }^{*}$ LI TAO, XIAN-YI LIN, YI-DONG YANG, SI-WEI TAN and BIN WU \\ Department of Gastroenterology, The Third Affiliated Hospital of Sun Yat-sen University, \\ Guangzhou, Guangdong 510630, P.R. China
}

Received August 3, 2017; Accepted April 19, 2018

DOI: 10.3892/ijo.2018.4419

\begin{abstract}
Cystatin SN (cystatin 1, CST1) is a member of the cystatin superfamily which inhibits the proteolytic activity of cysteine proteases. CST1 is a tumor biomarker that provides useful information for the diagnosis of esophageal, gastric and colorectal carcinomas. MicroRNAs (miRNAs or miRs) play an important role in tumor cell proliferation. However, the exact role of let-7d and CST1 in colon cancer remains unknown. The aim of this study was to assess whether let-7d inhibits colorectal carcinogenesis through the CST1/p65 pathway, and determine whether it may be used as a potential target for clinical therapy. Microarray analysis of mRNAs extracted from colon cancer and normal tissues was performed. The results of gene expression microanalysis revealed that CST1 expression was upregulated in colon cancer compared with normal tissues. In addition, the upregulation of CST1 expression and the downregulation of let-7d expression in patients with colon cancer and in several colorectal cancer cell lines were confirmed by reverse transcription-quantitative PCR (RT-qPCR), immunohistochemistry and western blot analysis. In addition, siRNA targeting CST1 (CST1-siRNA) and let-7d-mimics were used in the HCT116 cells, and the results revealed that CST1 and let-7d played a role in colorectal cancer cell proliferation. Let-7d inhibited colorectal carcinogenesis through the CST1/p65 pathway. Thus, the findings of the present study indicate that CST1 may be a potential target for the future clinical therapy of colorectal cancer.
\end{abstract}

Correspondence to: Professor Bin Wu, Department of Gastroenterology, The Third Affiliated Hospital of Sun Yat-sen University, 600 Tianhe Road, Guangzhou, Guangdong 510630, P.R. China

E-mail:wubin6@mail.sysu.edu.cn

*Contributed equally

Abbreviations: CST1, cystatin 1; miRNA or miR, microRNA; PCNA, proliferating cell nuclear antigen; DMEM, Dulbecco's modified Eagle's medium; NNF- $\kappa \mathrm{B}$, nuclear factor- $\kappa \mathrm{B}$

Key words: colon cancer, let-7d, cystatin 1, cell proliferation, p65

\section{Introduction}

Colon carcinogenesis is a multistep and complex process, involving the accumulation of a string of genetic and epigenetic alterations in normal colonic epithelial cells (1). Some of the frequent alterations found in colon cancer involve mutations in the tumor suppressors p53 and adenomatous polyposis coli $(A P C)$, as well as those in the oncogenes, $B R A F$, phosphoinositide 3 -kinase $(P I 3 K), K$-ras and $\beta$-catenin, some of which are closely associated with the control of cell proliferation (1). The overexpression of proliferation-associated proteins, such as leucine-rich repeat-containing G-protein coupled receptor 5 (Lgr5), EPH receptor B3 (EphB3) and Sox9 is also common in cancer (2-4). Overproliferation in the intestinal epithelium is a crucial mechanism deregulating the control of the turnover of intestinal epithelial cells, and is progressively incontrollable in colon carcinogenesis (2). Excessive proliferation is a hallmark of cancer, leading to an enhanced risk of cell self-clonal expansion, which in turn, increases the possibility of the occurrence of tumorigenic, including the accumulation of genetic mutations, angiogenesis, growth under stress conditions and an extended life span (5). Studies directly investigating the regulatory mechanisms of malignant cell proliferation are still largely and urgently required.

Cystatin 1 (CST1), a secretory peptide and a member of the type 2 cystatin superfamily, has been reported to be closely associated with cell proliferation and metastasis in several types of cancer, such as pancreatic malignant neoplasm, gastric cancer, esophageal squamous cell carcinoma and colorectal cancer (6-9). Our previous study using microarray analysis identified CST1 as one of the most highly expressed genes on the array in pancreatic cancer. Further experiments demonstrated that CST1 is a useful biomarker for pancreatic cancer cell proliferation (6). Moreover, it has been demonstrated that the upregulation of CST1 contributes to cell proliferation and the inhibition of cathepsin in gastric cancer; cathepsin is widely expressed in tissues and plays a role in cell proliferation, the immune response and tissue remolding (7). CST1 was believed to be an independent predictor of the 5-year survival rate of patients with surgically resected esophageal squamous cell carcinoma (8). Moreover, CST1 has been reported to be a novel biomarker of colorectal cancer (9). However, the detailed mechanisms responsible for the regulatory effects of CST1 on colon cancer cell proliferation remain to be elucidated. 
MicroRNAs (miRNAs or miRs) play an important role in tumor cell proliferation. With the dysregulation of miRNAs, the risk of carcinogenesis increases after Dicer 1, a critical enzyme needed for miRNA processing, is genetically manipulated $(10,11)$. Let-7d miRNA is an important family member of the let-7 miRNAs, which suppresses a multitude of oncofetal mRNAs and other pro-proliferative and/or pro-metastatic targets, such as high-mobility group AT-hook 2 (HMGA2), insulinlike growth factor 2 mRNA-binding protein 1 (IGF2BPl), $I G F 2 B P 2$ and nuclear receptor subfamily 6 group A member 1 (NR6A1) (12-15). A number of studies have demonstrated that let-7d plays pivotal role in the initiation and development of several malignant neoplasms. Let-7d has been proven to be negatively associated with pancreatic ductal adenocarcinoma, breast cancer, oral cancer, prostate cancer, and head and neck cancer (16-20). Moreover, some researchers demonstrated that let-7d inhibited glioblastoma multiforme cell proliferation and accelerated cell apoptosis in melanoma, colorectal, and ovarian cancer $(21,22)$. However, the association between let- $7 \mathrm{~d}$ and colorectal cancer has been rarely reported.

In the current study, we used microarrays to identify that CST1 is one of the most highly expressed mRNAs in colon cancer samples, compared with normal colon tissues, and we then further verified CST1 expression in more clinical samples by reverse transcription-quantitative PCR (RT-qPCR). We then detected 5 miRNAs (miR15b, miR-145, miR-126, miR-21 and let-7d), which are known as tumor-associated genes in cancer, including colon cancer and normal tissues (23-25). We found that $l e t-7 d$ expression was significantly decreased in colon cancer tissues and it was the only miRNA which was related to CST1 in colorectal cancer cell lines following transfection with miRNA mimics. We verified that CST1 and let-7d have an effect on colorectal cancer cell proliferation. We further identified the CST1/p65 pathway as the downstream pathway of let-7d in modulating colorectal cancer cell proliferation via the overexpression of let-7d or the silencing of CST1. The results of the current study suggest that the let-7d/CST1/p65 pathway is a novel transduction pathway found in colorectal cancer cell proliferation and that it may prove to be useful in the prevention and treatment of colorectal cancer.

\section{Materials and methods}

Tissue samples. Colon malignant neoplasm and matched non-tumor tissues from 10 patients were harvested during surgery from The Third Affiliated Hospital of Sun Yat-sen University (Guangzhou, China). The information of the patients is presented in Table I. Their diagnosis was dependent on pathological analysis. Matched non-tumor tissues were normal colon mucosa with biopsy tissues from the suspected patients examined using a colonoscope, which were confirmed pathological normality. The specimens were instantly frozen following excision and reserved at $-80^{\circ} \mathrm{C}$ for microarray, RT-qPCR, western blot analysis and immunohistochemical analysis. This study was approved by the Institutional Review Board at The Third Affiliated Hospital of Sun Yat-sen University. Written informed consent was obtained from each patient.

Microarray experiment. The four normal samples were normal colon mucosa with biopsy tissues from the suspected patients examined using a colonoscope. Six colon malignant neoplasm tissues were harvested during surgery. The samples were subsequently verified by histology assay. The microarray analysis was carried out by Capital Bio Corp. (Beijing, China). The array data were analyzed for data summarization, normalization and quality control using the GeneSpring software v12 (Agilent). The data were Log2 transformed and median centred by genes using the Adjust Data function of Cluster 3.0 software and then further analyzed with hierarchical clustering with average linkage.

Cell culture and transfection. The human colorectal cancer cell lines, including HCT116 (colon cancer), SW480 (colon adenocarcinoma), HT-29 (rectosigmoid adenocarcinoma) were purchased from the American Type Culture Collection (ATCC, Manassas, VA, USA). The cells were cultured in Dulbecco's modified Eagle's medium (DMEM; Gibco-BRL, New York, NY, USA) supplemented with $10 \%$ heat-inactivated fetal bovine serum and incubated in a humidified condition at $37^{\circ} \mathrm{C}$ with $5 \% \mathrm{CO}_{2}$. For overexpression or knock down experiments, 3 siRNAs targeting CST1 (20 $\mu \mathrm{M}$ CST1 siRNA; oligo kit; GenePharma, Shanghai, China), the let-7d mimic and inhibitor kit (GenePharma) and miR15b, miR-145, miR-126, miR-21 mimic kit (GenePharma) were transfected into the colorectal cancer cell lines using Lipofectamine 3000 (1901443, Invitrogen, Carlsbad, CA, USA) according to the manufacturer's instructions; the sequences of siRNAs and mimics are listed in Table II. The most effective siRNA sequence (number 3, Table II; the results of the most effective siRNA are not shown) was screened to attain a transfection efficiency of $>90 \%$ and was selected to be used in the experiments. Following 1 day of incubation, the transfection medium with Lipofectamine 3000 (Invitrogen) was discarded and regular culture medium was added for incubation in a humidified atmoshpere at $37^{\circ} \mathrm{C}$ with $5 \% \mathrm{CO}_{2}$. At $48 \mathrm{~h}$ after transfection, the human colorectal cancer cell lines were used in further experiments. Subsequently, Bay117082 (B5556; Sigma, St. Louis, MO, USA), an NNF-кB inhibitor was added at an optimal dose of $30 \mu \mathrm{mol} / 1$ into the medium of HCT116 cells for $2 \mathrm{~h}$ before further experiments.

RNA extraction and $R T-q P C R$. With the RNAgents Total RNA Isolation system (Promega, Madison, WI, USA), total RNA was extracted from the cancer tissues, matched normal tissues and cell lines according to the manufacturer's instructions. For miRNA quantification, with primer sets (GenePharma), total RNA was reverse transcribed into cDNA for quantitative PCR. U6 was used to normalize the expression of let-7d. First-strand cDNA was synthesized using Superscript Reverse Transcriptase (Invitrogen) following the manufacturer's instructions. cDNA was diluted with diethyl pyrocarbonate-treated water at a 1:10 ratio. Quantitative PCR was executed using specific primers and the QuantiTest SYBR-Green PCR kit (Cat. no. 04707516001) according to the manufacturer's instructions (Roche, Basel, Switzerland) on an Applied Biosystems 7500 real-time PCR machine (Applied Biosystems, Foster City, CA, USA). The mRNA primers used and the sequences were as follows: CST1 sense, 5'-GGTACAG CGTGCCCTTCA-3' and antisense, 5'-TTGGGCTGGGACT TGGTA-3', 171-bp product; proliferating cell nuclear antigen $(P C N A)$ sense, 5'-CCTGCTGGGATATTAGCTCCA-3' and 
Table I. The clinical characteristics of the patients.

\begin{tabular}{|c|c|}
\hline Clinicopathology and characteristic & Total \\
\hline Total, $\mathrm{n}$ & 10 \\
\hline Age $($ mean $\pm \mathrm{SD})$ & $58 \pm 8.5$ \\
\hline \multicolumn{2}{|l|}{ Age, n $(\%)$} \\
\hline$<65$ & 2 \\
\hline$\geq 65$ & 8 \\
\hline \multicolumn{2}{|l|}{ Sex } \\
\hline Male & 4 \\
\hline Female & 6 \\
\hline \multicolumn{2}{|l|}{ Tumor location } \\
\hline Right colon & 7 \\
\hline Left colon & 3 \\
\hline \multicolumn{2}{|l|}{$\mathrm{T}$ category } \\
\hline pT1 & 1 \\
\hline pT2 & 2 \\
\hline pT3 & 4 \\
\hline pT4 & 3 \\
\hline \multicolumn{2}{|l|}{$\mathrm{N}$ category } \\
\hline 0 & 3 \\
\hline 1 & 4 \\
\hline 2 & 3 \\
\hline \multicolumn{2}{|l|}{ Distant metastasis } \\
\hline Absent & 6 \\
\hline Present & 4 \\
\hline \multicolumn{2}{|l|}{ Differentiation } \\
\hline Well/moderate & 6 \\
\hline Poor & 4 \\
\hline
\end{tabular}

antisense, 5'-CAGCGGTAGGTGTCGAAGC-3', 109-bp product; cyclin Dl sense, 5'-GCTGCGAAGTGGAAACC ATC-3' and antisense, 5'-CCTCCTTCTGCACACATTT GAA-3', 135-bp product; cyclin E sense, 5'-CCTGCGCGAGA AGGAACTG-3' and antisense, 5'-CGTTGTAGCGATCCAT GAAGTG-3', 173-bp product; and $\beta$-actin sense, 5'-GTCTTCC CCTCCATCGTG-3' and antisense, 5'-AGGGTGAGGATGC CTCTCTT-3', 113-bp product. The reaction conditions were as follows: $95^{\circ} \mathrm{C}, 5 \mathrm{~min} ;\left(95^{\circ} \mathrm{C}, 15 \mathrm{sec} ; 60^{\circ} \mathrm{C}, 30 \mathrm{sec}\right) \mathrm{x} 40$ cycles. mRNA expression was normalized to $\beta$-actin.

Immunohistochemistry. Formalin-fixed tissues were embedded in paraffin and sliced. The 4-mm-thick sections were then incubated with monoclonal antibodies against CST1 (1:100, Sigma) overnight at $4^{\circ} \mathrm{C}$ and then incubated with goat anti-rabbit secondary antibodies (sc-2006, Santa Cruz Biotechnology, Santa Cruz, CA, USA). 3,3'-Diaminobenzidine was used as the chromogen. The sections were then dyed using hematoxylin and mounted. CST1 expression was evaluated qualitatively by two independent pathological experts unaware of the clinical and pathological information.

Western blot analysis. Total protein was extracted from the tissues and cell lines using tissue extraction reagent (FNN0071,
Table II. Sequences of siRNAs and mimics used.

\begin{tabular}{lr}
\hline Gene & Sequence \\
\hline CST1 siRNA & 1. 5 5'-GGUACUAAGAGCCAGGCAATT-3' \\
& 5'-UUGCCUGGCUCUUAGUACCTT-3' \\
& 2. 5'-GGUGGCAUCUAUAACGCAGTT-3' \\
& 5'-CUGCGUUAUAGAUGCCACCTT-3' \\
& 3. 5'-GCCAUCAGCGAGUAUAACATT-3' \\
& 5'-UGUUAUACUCGCUGAUGGCTT-3' \\
Let-7d mimic & 5'-AGAGGUAGUAGGUUGCAUAGUU-3' \\
& 5'-AACUAUGCAACCUACUACCUCU-3' \\
miRNA15b & 5'-UAGCAGCACAUCAUGGUUUACA-3' \\
& 5'-UGUAAACCAUGAUGUGCUGCUA-3' \\
miR-145 & 5'-GUCCAGUUUUCCCAGGAAUCCCU-3' \\
& 5'-AGGGAUUCCUGGGAAAACUGGAC-3' \\
miR-126 & 5'-CAUUAUUACUUUUGGUACGCG-3' \\
& 5'-CGCGUACCAAAAGUAAUAAUG-3' \\
miR-21 & 5'-UAGCUUAUCAGACUGAUGUUGA-3' \\
& 5'-UCAACAUCAGUCUGAUAAGCUA-3' \\
\hline
\end{tabular}

Invitrogen) and cell extraction buffer (FNN0011, Invitrogen), purified and assessed qualitatively by western blot analysis. Total protein extracts $(40 \mu \mathrm{g})$ were shifted to $10 \%$ gradient SDS-PAGE gels to separate the proteins by different molecular weight and transferred onto nitrocellulose membranes for antigen-antibody reaction. The membranes were then blocked with 5\% skimmed milk in PBS for $2 \mathrm{~h}$ and mixed with primary antibodies against CST1 (SAB-1405670, Sigma), p-p65 (sc-101749), PCNA (sc-56), cyclin D1 (sc-753), p65 (sc-372) (Santa Cruz Biotechnology) and $\beta$-actin (sc-47778, Santa Cruz Biotechnology) and then incubated with goat anti-rabbit secondary antibodies (sc-2006, Santa Cruz Biotechnology) for $2 \mathrm{~h}$ at $37^{\circ} \mathrm{C}$.

Colony formation assay. To qualitatively evaluate clonogenicity, the HCT-116 cells were transfected with siRNA against CST1 (CST1-siRNA; GenePharma), let-7d mimic or the control empty vector (GenePharma) for $48 \mathrm{~h}$ and the HCT-116 cells were then cultured in 6 -well plates at a density of 200 cells/well. The 6-well plates were then washed with PBS, fixed in methanol for $15 \mathrm{~min}$, and stained with $0.5 \%$ crystal violet for $15 \mathrm{~min}$. The plates were then photographed, and the colonies were counted with a high-resolution microscope (DMI 3000, Leica, Wetzlar, Germany).

Statistical analysis. All statistical analyses were performed using SPSS 18.0 software. Paired t-tests were executed to compare CST1 and let-7d expression among the sample tissues and cell lines. Significant differences were considered if the probability of the difference was $<5$ in $100(\mathrm{P}<0.05)$.

\section{Results}

Upregulation of CST1 in patients with colon cancer. In the current study, we used GeneChip microarrays to compare 


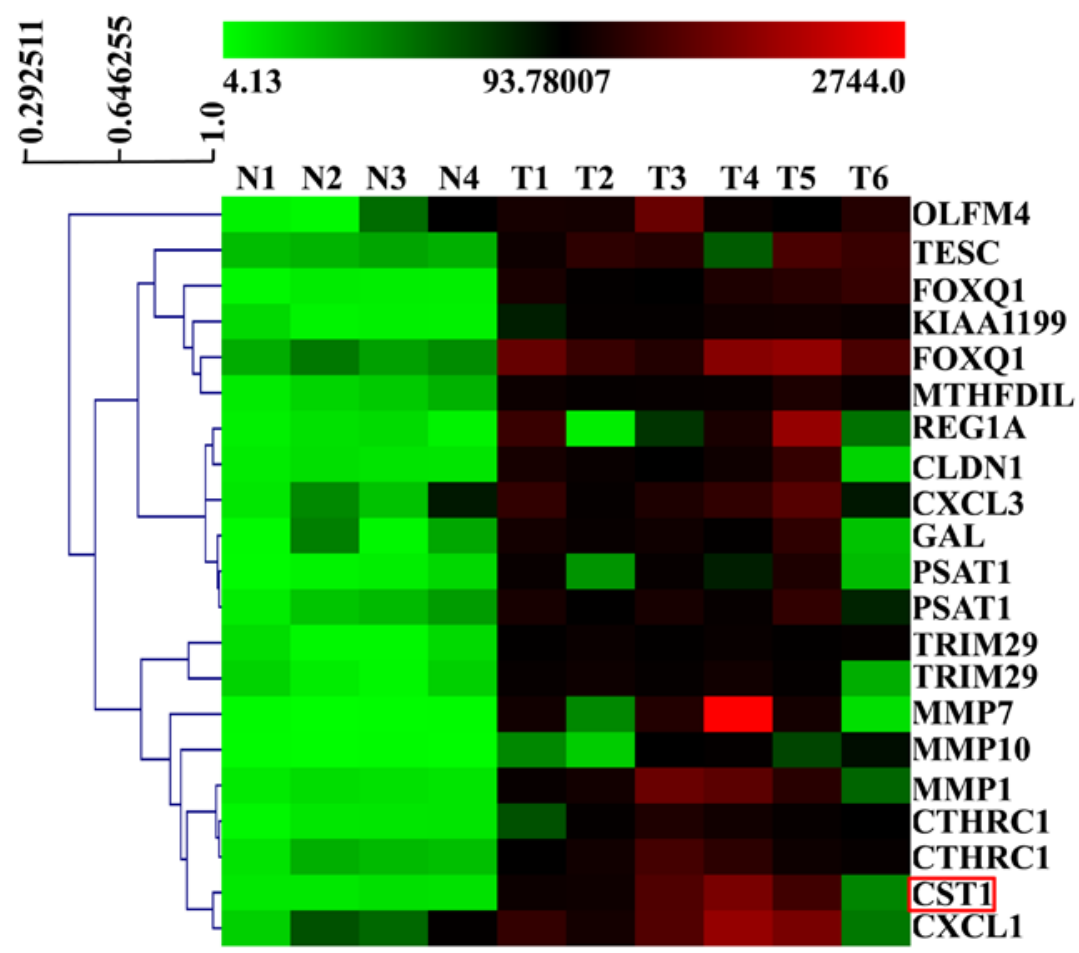

Figure 1. Microarray results of human colon cancer tissues and non-cancerous tissues. CST1 expression was conspicuously increased in cancer tissues compared with normal colon tissues in the microarray. CST1 is indicated in a red square. ' $\mathrm{N}$ ' indicates normal tissue and ' $\mathrm{T}$ ' indicates tumor tissue.

the RNA expression profiles of 6 colorectal cancer tissues and 4 normal tissues. The microarrays revealed that CSTI expression was significantly increased in the cancer samples compared with the normal tissues (Fig. 1). This indicated that CST1 may be involved in the development and/or progression of colon cancer.

Expression of miRNAs in human colorectal cancer. We analyzed the expression of miR15b, miR-145, miR-126, miR-21 and let-7d in 5 cancer tissues and 5 normal tissues by RT-qPCR. The results revealed that let-7d expression was significantly decreased by approximately 20 -fold in the cancer tissues (Fig. 2A). We then used different miRNA mimics to increase the expression of 5 miRNAs in the HCT116 cells and examine the effects on miRNA expression. The results revealed that CST1 expression was markedly decreased when using let-7d-mimic (Fig. 2B). Thus, our findings indicate that let-7d expression was decreased in the colon cancer tissues and hypothesized that it was related to CST1.

Upregulation of CST1 in human colon cancer. To confirm that CST1 was upregulated in the colon cancer tissues, we verified its mRNA expression in the colon cancer tissues and normal tissues by RT-qPCR. CST1 mRNA expression was found to be markedly increased by $\sim 5$-fold in the cancer compared with the normal tissues, consistent with the results of the microarray analysis (Fig. 3A). Moreover, western blot analysis revealed that CST1 protein expression was also highly upregulated in the cancer tissues, but downregulated in the normal tissues (Fig. 3B and C). Furthermore, by using immunohistochemistry, CST1 protein expression was found to be increased conspicuously in the tumor tissues, but not

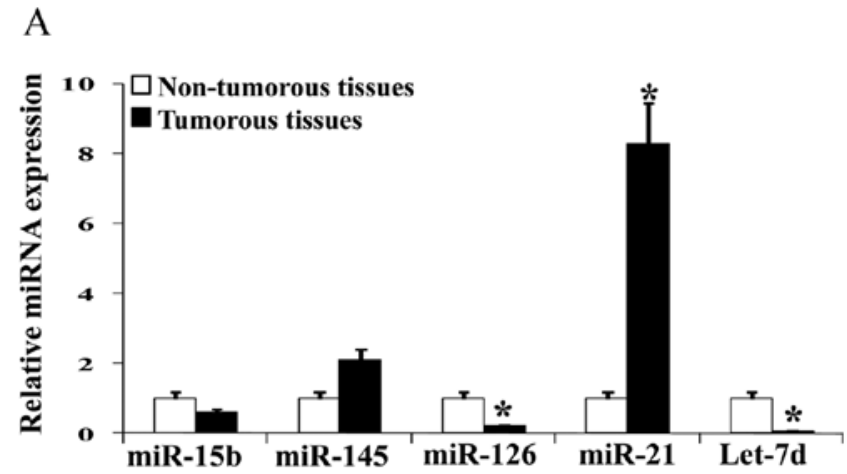

B

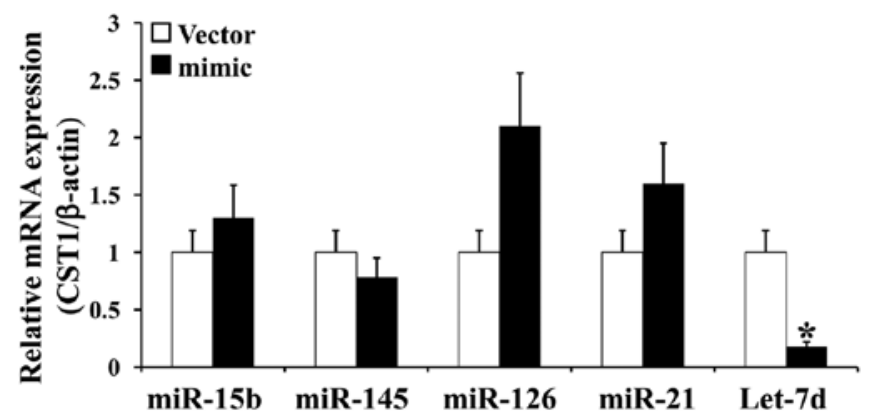

Figure 2. Expression of miRNAs in human colon cancer. Whole RNA was extracted from 5 patients with colon cancer and 5 with non-colon cancer and PCR analysis was performed. Each cDNA generated from non-tumorous (N) and tumorous (T) tissues was used to amplify miRNAs. (A) The expression level of 5 miRNAs was measured as the relative ratio of miRNA to $U 6$ in each sample ( ${ }^{*} \mathrm{P}<0.05$ vs. normal tissues). (B) The human colon cancer cell line, HCT116, was used and was transfected with let-7d-mimics or the negative control (NC). The expression level of CST1 was expressed as the relative ratio of CST1 to $\beta$-actin in each sample $\left({ }^{*} \mathrm{P}<0.05\right.$ vs. normal tissues). 
A

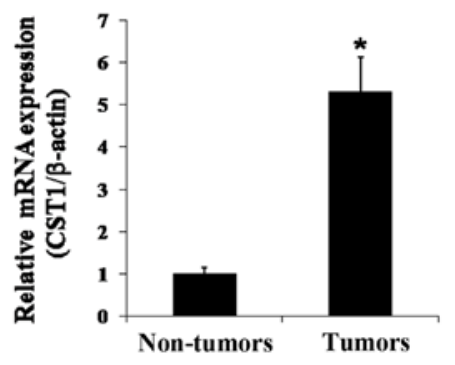

B

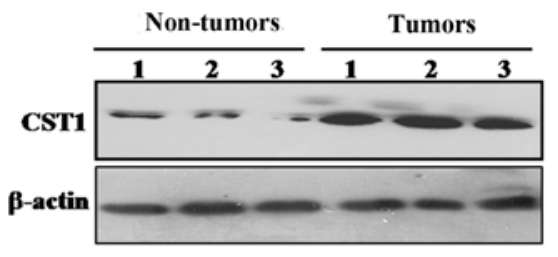

C

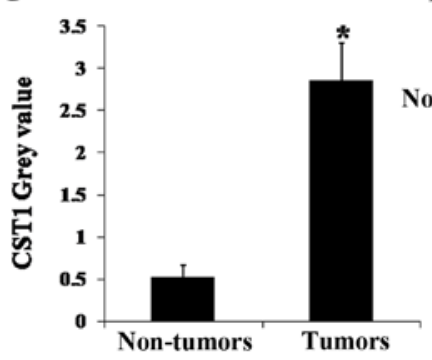

D

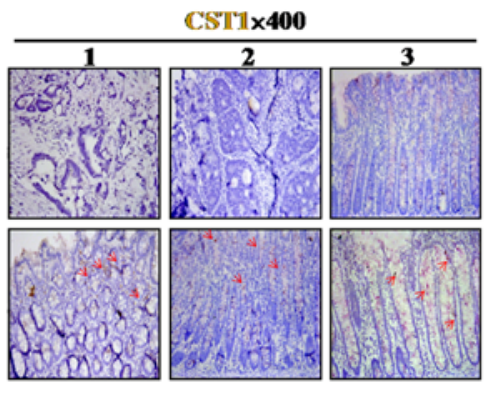

Figure 3. Upregulation of CST1 in human colon cancer. cDNA generated from 5 non-tumorous (N) and 5 tumorous (T) tissues was used to amplify CST1. (A) The expression level of CST1 was measured as the relative ratio of CST1 to $\beta$-actin in each sample ("P<0.05 vs. normal tissues). (B) A total of 3 tumorous and 3 non-tumorous tissues were evaluated by western blot analysis to investigate CST1 expression. (C) The grey value of CST1 protein was assessed ("P $<0.05$ vs. normal tissues). (D) Patient samples were dyed with CST1 primary antibody. CST1 expression was conspicuously increased in tumorous tissues. The red arrows indicate CST1-positive signals.

A

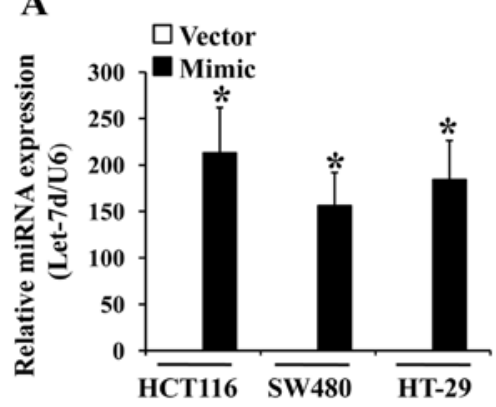

C
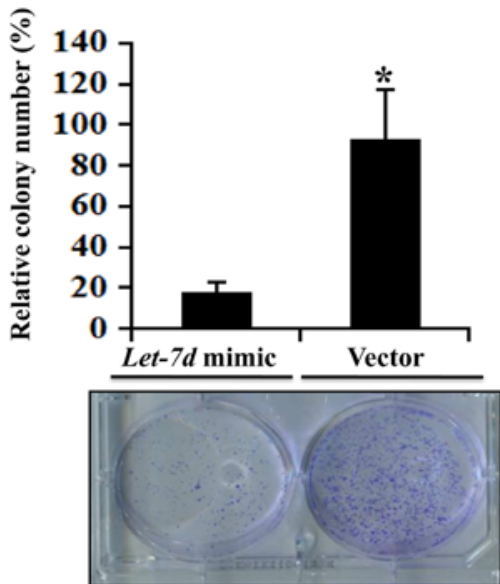

B

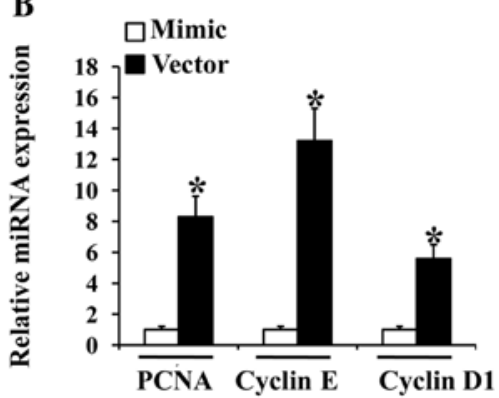

D

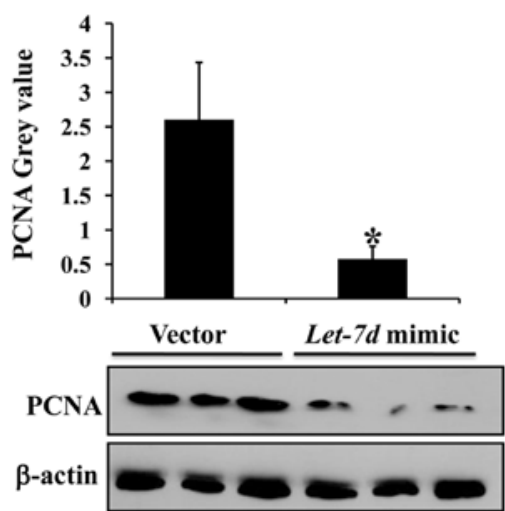

Figure 4. Overexpression of let-7d suppresses colon cancer cell proliferation. Human colorectal cancer cell lines (HCT116, SW480 and HT-29) were used and transfected with let-7d-mimics or the negative control (NC). Total RNA was extracted and RT-qPCR analysis was performed. (A) Transfection with let-7d-mimics markedly enhanced let-7d expression in the colorectal cancer cell lines, as shown by RT-qPCR. The expression level of let-7d was measured as the relative ratio of let-7d to U6 in each sample ("P $<0.05$ vs. control vector group). (B) Amplification of PCNA, cyclin E and cyclin D1 in HCT116 cells transfected with let-7d-mimics or the negative control (NC). Each proliferation-associated proteins was expressed as the relative ratio to $\beta$-actin ("P<0.05 vs. control vector group). (C) Representative images of colony formation of HCT116 cells transfected with NC or let-7d-mimics (" $\mathrm{P}<0.05$ vs. control vector group). (D) Expression of the proliferation-associated protein, PCNA, examined by western blot analysis, indicating that CST1 may regulate colorectal cancer cell proliferation ("P<0.05 vs. control vector group). 

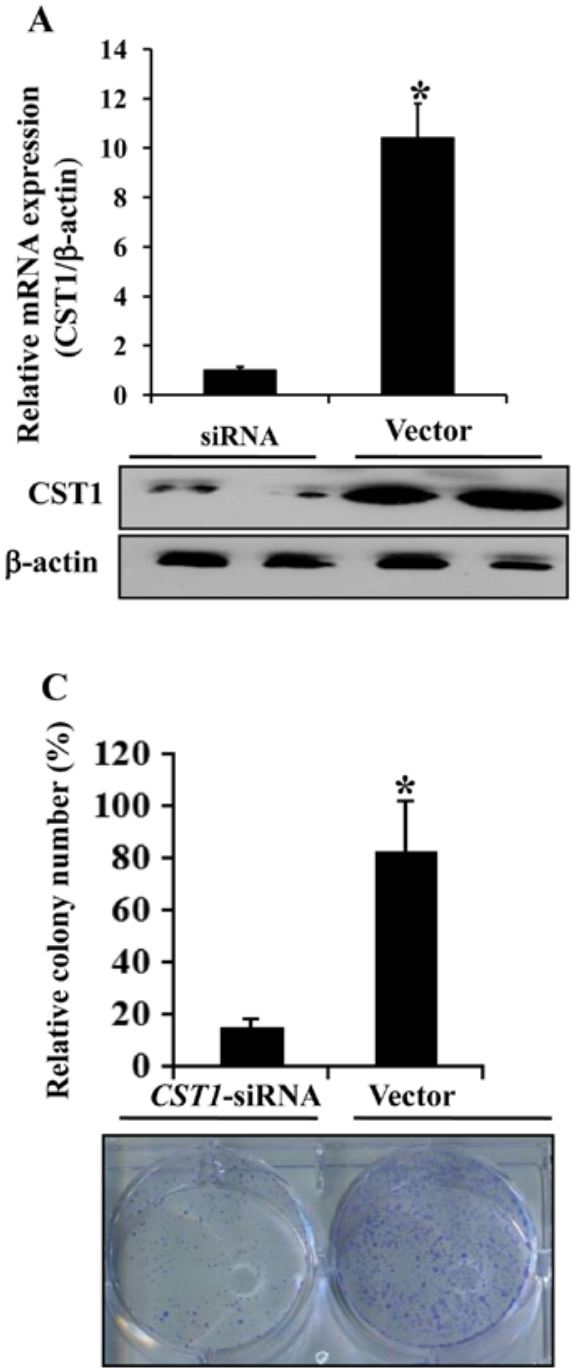

B

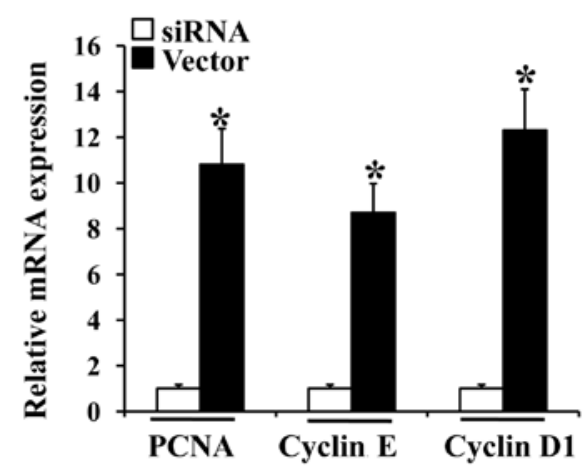

D
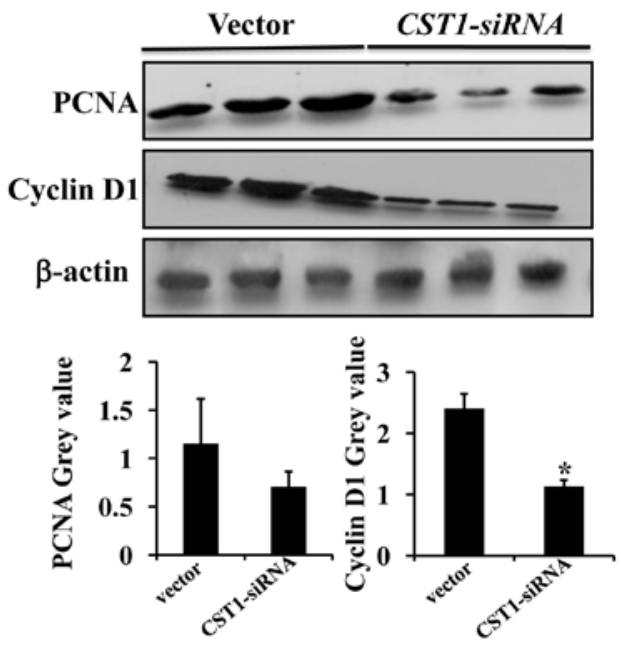

Figure 5. CST1 contributes to human colon cancer cell proliferation. HCT116 cells were transfected with CST1-siRNA or the negative control (NC). (A) CST1-siRNA notably decreased CST1 expression in the colon cancer cell line, as shown by RT-qPCR and western blot analysis. The expression level of CST1 was measured as the relative ratio of $C S T 1$ to $\beta$-actin in each sample (" $\mathrm{P}<0.05$ vs. control vector group). (B) Amplification of $P C N A$, cyclin $E$ and cyclin D1 in HCT116 cells transfected with CST1-siRNA or the negative control (NC). The expression level of each was expressed as the relative ratio to $\beta$-actin ("P $\mathrm{P}<0.05$ vs. control vector group). (C) Representative images of colony formation of HCT116 cells transfected with CST1-siRNA or NC ("P $<0.05$ vs. control vector group). (D) Expression of the proliferation-associated proteins, PCNA and cyclin D1, examined by western blot analysis, revealing that CST1 may participate in colorectal cancer cell proliferation (" $\mathrm{P}<0.05$ vs. control vector group).

in the normal tissues (Fig. 3D). These data thus suggest that CST1 plays a role in the initiation and development of color cancer.

Overexpression of let-7d suppresses colorectal cancer cell proliferation. We screened the most efficient and effective let-7d mimics for upregulating let-7d expression for use in the subsequent experiments (Fig. 4A). The mRNA expression levels of cancer proliferation-associated proteins, such as PCNA, cyclin D1 and cyclin E, were markedly diminished in the HCT116 cells transfected with let-7d-mimics (Fig. 4B). Colony formation assays were executed using the HCT116 cells transfected with let-7d-mimics or the empty vector and found that compared with the controls, the mean colony number was significantly decreased in the group transfected with let-7d-mimics (Fig. 4C). Combined with the decrease in the expression of the proliferation-associated protein, PCNA, in the group transfected with let-7d-mimics
(Fig. 4D), it was suggested that let-7d regulates colorectal cancer cell proliferation.

CST1 contributes to human colorectal cancer cell proliferation. We screened the most efficient and effective CSTI-siRNA from 3 siRNAs for inhibiting CST1 expression for use in the subsequent experiments (Fig. 5A). The mRNA expression levels of the cancer proliferation-associated proteins, PCNA, cyclin D1 and cyclin E, were markedly decreased in the HCT116 cells transfected with CST1-siRNA compared with the cells transfected with the control vector (Fig. 5B). The results of colony formation assay revealed that compared with the control, the mean colony number was significantly decreased in the HCT116 cells transfected with CST1-siRNA (Fig. 5C). We then evaluated the expression of proliferationassociated proteins and found that the expression of PCNA and cyclin D1 was markedly decreased in the HCT116 cells transfected with CST1-siRNA (Fig. 5D). Thus, these findings 

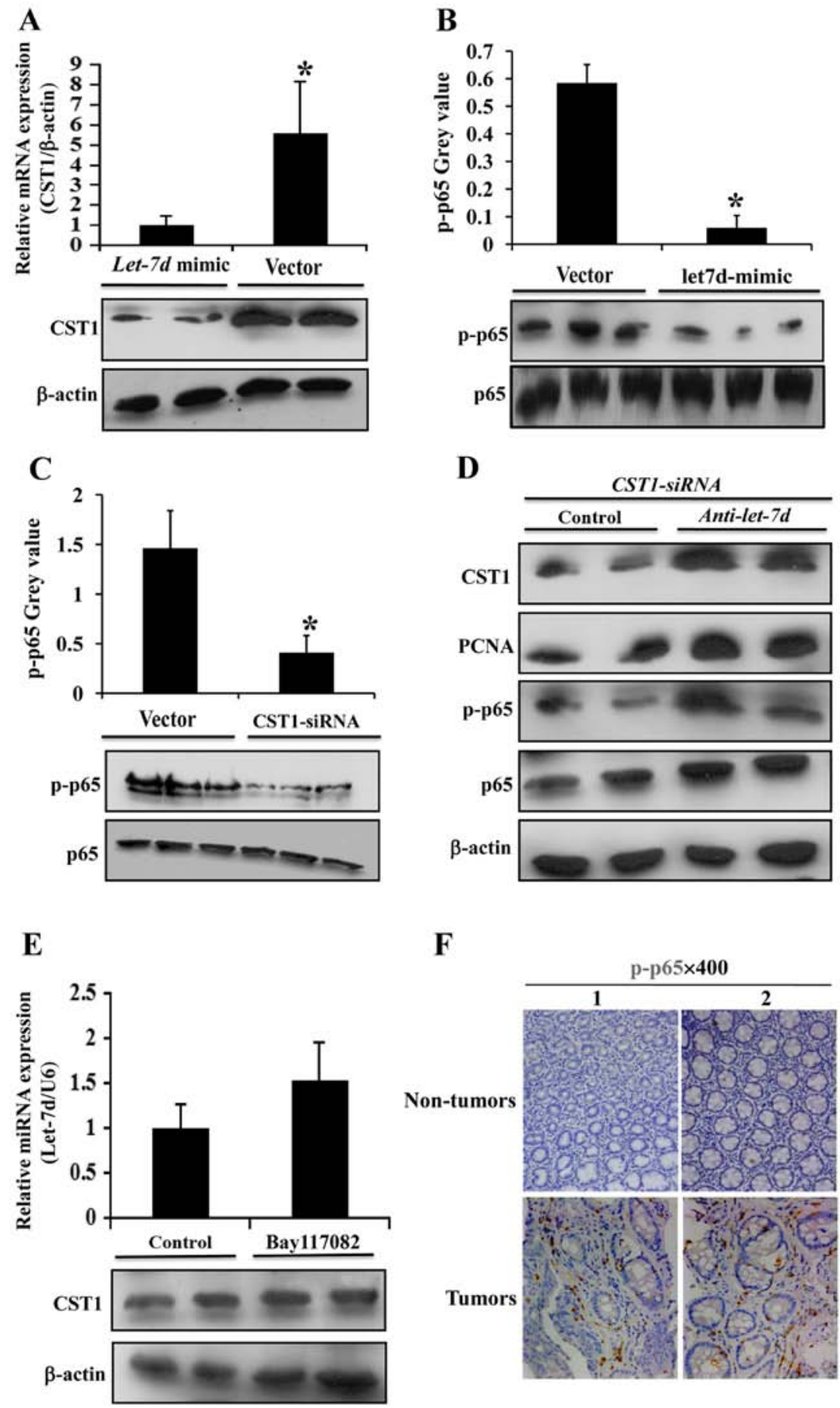

Figure 6. Let-7d inhibits colorectal cancer cell proliferation through CST1/p65. HCT116 cells were transfected with CST1-siRNA, let-7d-mimics or the negative control (NC). (A) CST1 expression significantly decreased in the HCT116 cells transfected with let-7d-mimics, as shown by RT-qPCR and western blot analysis. The expression level of CST1 was calculated as the relative ratio of CST1 to $\beta$-actin in each sample ("P $<0.05$ vs. control vector group). (B) NNF- $\mathrm{kB}$ p65 phosphorylation was examined by western blot analysis following transfection with let- $7 d$-mimics ("P $<0.05$ vs. control vector group). (C) NNF- $\mathrm{B}$ p65 phosphorylation was examined by western blot analysis following transfection with CST1-siRNA ("P<0.05 vs. control vector group). (D) The downregulation of CST1, PCNA and p-p65 which was induced by transfection with CST1-siRNA was abrogated by the use of anti-let-7d in the HCT116 cells. (E) Expression of let-7d and CST1 following treatment of the cells with Bay117082 (an NF- $\mathrm{kB}$ p65 inhibitor). (F) Tissues from patients with colon cancer were stained with p-p65 antibody. NNF- $\mathrm{BB}$ expression was increased in the tumorous tissues.

suggest that CST1 promotes colorectal cancer cell proliferation by increasing PCNA and cyclin D1 expression.

Let-7d inhibits colorectal cancer cell proliferation through CST1/p65. We used let-7d-mimics to increase let-7d expression in the HCT116 cells and we found that in this process, CST1 expression was markedly decreased (Fig. 6A). We also used CSTI-siRNA to silence CST1 expression in the HCT116 cells; however, the silencing of CST1 had no marked effect on let-7d expression (data not shown); this indicated that let-7d may regulate CST1 expression in colorectal cancer. NNF- $\kappa \mathrm{B}$ p65 phosphorylation was then analyzed, and it was found to decrease following transfection with let-7d-mimics (Fig.6B), indicating that let-7d may be connected with the NNF- $\mathrm{KB}$ 
p65 pathway. Subsequently, following the silencing of CST1, NNF- $\kappa \mathrm{B}$ p65 phosphorylation was analyzed, and it was found to markedly decrease (Fig. 6C). Moreover, following the use of let-7d inhibitor (anti-let-7d) in the HCT116 cells, the downregulation of CST1, PCNA and p-p65 observed with the CSTI-siRNA was abrogated (Fig. 6D). In addition, we used Bay117082 (a NNF- $\mathrm{B}$ p65 inhibitor) in the HCT116 cells; it was not found to have any marked effect on let-7d and CST1 expression (Fig. 6E). The results of immunohistochemistry confirmed that p-p65 expression was upregulated in the human colon cancer tissues (Fig. 6F), compared with the normal tissues. Thus, the above-mentioned data demonstrate that the inhibitory effects of let-7d on colorectal cancer progression are partly mediated by the targeting of CST1 via NNF-кB p65.

\section{Discussion}

Colon cancer is one of the most malignant types of cancer; however, the molecular mechanisms that underlie the development of colon cancer remain unclear. Let-7 miRNAs comprise one of the largest and most highly expressed families of miRNAs, possessing potent anti-carcinogenic properties in a variety of tissues (12). Although a number of studies have demonstrated that let-7d plays a pivotal role in the initiation and development of several malignant neoplasms, the association between let-7d and colon cancer has rarely been reported. Madison et al reported that let-7d was negatively associated with intestinal cancer and that let-7 suppressed carcinogenesis and the stem cell phenotypic regulation of Hmga2 (26). The transcription factor, NNF- $\kappa \mathrm{B}$, a master regulator of cell survival, inflammation and immunity, has been shown to comprise a key link between inflammation and cancer (27). The association between let-7d and NNF- $\mathrm{kB}$ p65 has not yet been reported, at least to the best of our knowledge; our study thus focused on the mechanisms of action of let-7d in colon carcinogenesis via NNF- $\mathrm{kB}$ p65.

Cysteine proteases, including cathepsins and papain are proteolytic enzymes that are highly expressed in different tissues and have been found to participate in several pathophysiological procedures, such as tissue reconstitution, inflammatory tissue damage, regulation of the immune response and the migration of monocytes and cancer cells (28-30). The cystatin (CST) superfamily function as inhibitors in the proteolytic activity of cysteine proteases by constituting tight, but reversible complexes $(31,32)$. Previous studies have revealed that cystatins exert a significant effect on malignant tumor invasion and metastasis. The type 2 cystatin superfamily contains CST1, CST2, CST3, CST4, CST5, CSTP1, CSTP2 and CST1 (CST SN).

Moreover, CST2 (CST SA) and CST4 (CST S) are known as S-type cystatins. CST1 encoding CST SN is a member of type 2 cystatins, which are highly expressed in the uterus, submandibular gland and gallbladder, but not in the colon, according to previous findings (33). Our previous study indicated that CST1 expression was highly elevated in pancreatic cancer, contributing to pancreatic cancer cell proliferation, and was a potential biomarker for the early detection of pancreatic cancer (6). Previous studies have found that CST1 plays a critical role in gastric cancer, colorectal cancer and esophageal squamous cell carcinoma (6-9). An elevated CST1 expression in colorectal cancer was found to contribute to colorectal carcinogenesis by counteracting the inhibitory effects of CST3 on the proteolytic activity of cathepsin B $(9,34)$. The association between let-7d and the CST1/p65 pathway has not yet been reported, at least to the best of our knowledge. Thus, our study focused on the mutual regulation of let-7d and CST1 in colon cancer, with an aim to explore a novel mechanism of tumor cell proliferation in colon carcinogenesis.

In the current study, our microarrays comparing gene expression in colon cancer and control tissues indicated that CSTl was one of the most highly and conspicuously expressed of the 22,000 genes on the array (Fig. 1) and we then verified CST1 mRNA and protein expression in colon cancer tissues compared with non-cancer control tissues and the results were consistent with those of the microarray. CST1 was upregulated at both the mRNA and protein level (Fig. 3). We found that let-7d expression was downregulated in the colon cancer tissues compared with the non-cancer controls (Fig. 2A) and it was the only miRNA that had an effect on CST1 expression when using miRNA-mimics among the 5 miRNAs (miR $15 \mathrm{~b}$, miR-145, miR-126, miR-21 and let-7d) (Fig. 2B). We further used let-7d-mimic to increase its expression in the colorectal cancer cell lines, HCT116, SW480 and HT-29 (Fig. 4A). In addition, let- $7 d$-mimic was used to increase let-7d expression in the HCT-116 cells (Fig. 4C), and we found that let-7d overexpression suppressed the expression of proliferationassociated proteins, such as PCNA, cyclin D1 and cyclin E (Fig. 4B) suggesting that let-7d may be involved in colorectal cancer cell proliferation. The suppression of CST1 by CST1-siRNA in the HCT116 cells in colony formation assays revealed that CST1 participates in colorectal cancer cell proliferation (Fig. 5A and C). Consistent with this finding, CST1 silencing decreased the expression of malignancyassociated proteins, such as PCNA, cyclin D1 and cyclin E (Fig. 5B and D). On the basis of the results mentioned above, we hypothesized that maybe let-7d was associated with CST1 and played a role in colon carcinogenesis. However, we then wished to determine the pathway involved. We further used let-7d mimic to increase its expression in the HCT116 cells, and found that CST1 expression decreased significantly (Fig. 6A). A high expression of let-7d inhibited NNF-кB p65 phosphorylation (Fig. 6B) and the silencing of CST1 also inhibited NNF- $\mathrm{kB}$ p65 phosphorylation (Fig. 6C). We then silenced CST1, and also used let-7d inhibitor in the HCT116 cells; the use of let-7d inhibitor abrogated the suppressive effects of the silencing of CST1 on CST1, PCNA and p-p65 expression (Fig. 6D). We also used Bay117082 (an NNF-kB p65 inhibitor) in the HCT116 cells, and it found to have no marked effect on let-7d and CST1 expression (Fig. 6E). Finally, our immunohistochemical analysis confirmed that p-p65 expression was upregulated in the human colon cancer tissues (Fig. 6F).

In conclusion, this study demonstrates that the upregulation of CST1 in colorectal cancer contributes to cell proliferation. Let-7d inhibits colorectal carcinogenesis through the CST1/ p65 pathway, and may thus be prove to be a potential target for clinical therapy. The results of this study were obtained from in vitro experiments. In the future, we aim to examine the 
effects of let-7d on colorectal cancer proliferation and growth in vivo in order to confirm the findings of this study.

\section{Acknowledgements}

The authors would like to express their gratitude for the helpful comments on this manuscript received by the reviewers.

\section{Funding}

This study was partly supported by grants from the National Natural Science Foundation of China (nos. U1501224 and 81370511), and the Natural Science Foundation of Guangdong Province (no. S2013010016541).

\section{Availability of data and materials}

All data generated or analyzed during this study are included in this published article.

\section{Authors' contributions}

$\mathrm{JJ}$ and HLL were the major contributors in performing the experiments and the writing of the manuscript. LT assisted in performing the experiments. XYL provided technical assistance. YDY and SWT provided assistance with the collection of the clinical samples, as well as technical assistance. BW was involved in the design of the study, and was also in charge of our laboratory. All authors have read and approved the final manuscript.

\section{Ethics approval and consent to participate}

This study was approved by the Institutional Review Board at The Third Affiliated Hospital of Sun Yat-sen University. Written informed consent was obtained from each patient.

\section{Consent for publication}

Not applicable.

\section{Competing interests}

The authors declare that they have no competing interests.

\section{References}

1. Vogelstein B and Kinzler KW: Cancer genes and the pathways they control. Nat Med 10: 789-799, 2004.

2. Schwitalla S, Fingerle AA, Cammareri P, Nebelsiek T, Göktuna SI, Ziegler PK, Canli O, Heijmans J, Huels DJ, Moreaux G, et al: Intestinal tumorigenesis initiated by dedifferentiation and acquisition of stem-cell-like properties. Cell 152: 25-38, 2013.

3. Li G, Ji XD, Gao H, Zhao JS, Xu JF, Sun ZJ, Deng YZ, Shi S, Feng YX, Zhu YQ, et al: EphB3 suppresses non-small-cell lung cancer metastasis via a PP2A/RACK1/Akt signalling complex. Nat Commun 3: 667, 2012.

4. Hessmann E, Zhang JS, Chen NM, Hasselluhn M, Liou GY, Storz P, Ellenrieder V, Billadeau DD and Koenig A: NFATc4 regulates Sox9 gene expression in acinar cell plasticity and pancreatic cancer initiation. Stem Cells Int 2016: 5272498, 2016.

5. Hanahan D and Weinberg RA: The hallmarks of cancer. Cell 100: $57-70,2000$
6. Jiang J, Liu HL, Liu ZH, Tan SW and Wu B: Identification of cystatin $\mathrm{SN}$ as a novel biomarker for pancreatic cancer. Tumour Biol 36: 3903-3910, 2015.

7. Choi EH, Kim JT, Kim JH, Kim SY, Song EY, Kim JW, Kim SY, Yeom YI, Kim IH and Lee HG: Upregulation of the cysteine protease inhibitor, cystatin SN, contributes to cell proliferation and cathepsin inhibition in gastric cancer. Clin Chim Acta 406: 45-51, 2009.

8. Chen YF, Ma G, Cao X, Luo RZ, He LR, He JH, Huang ZL, Zeng MS and Wen ZS: Overexpression of cystatin SN positively affects survival of patients with surgically resected esophageal squamous cell carcinoma. BMC Surg 13: 15, 2013.

9. Yoneda K, Iida H, Endo H, Hosono K, Akiyama T, Takahashi H, Inamori M, Abe Y, Yoneda M, Fujita K, et al: Identification of Cystatin SN as a novel tumor marker for colorectal cancer. Int J Oncol 35: 33-40, 2009.

10. Kumar MS, Pester RE, Chen CY, Lane K, Chin C, Lu J, Kirsch DG, Golub TR and Jacks T: Dicer1 functions as a haploinsufficient tumor suppressor. Genes Dev 23: 2700-2704, 2009.

11. Lambertz I, Nittner D, Mestdagh P, Denecker G, Vandesompele J, Dyer MA and Marine JC: Monoallelic but not biallelic loss of Dicer1 promotes tumorigenesis in vivo. Cell Death Differ 17: 633-641, 2010.

12. Büssing I, Slack FJ and Grosshans H: let-7 microRNAs in development, stem cells and cancer. Trends Mol Med 14: 400-409, 2008.

13. Lee YS and Dutta A: The tumor suppressor microRNA let-7 represses the HMGA2 oncogene. Genes Dev 21: 1025-1030, 2007.

14. Boyerinas B, Park SM, Shomron N, Hedegaard MM, Vinther J, Andersen JS, Feig C, Xu J, Burge CB and Peter ME: Identification of let-7-regulated oncofetal genes. Cancer Res 68: 2587-2591, 2008.

15. Gurtan AM, Ravi A, Rahl PB, Bosson AD, JnBaptiste CK, Bhutkar A, Whittaker CA, Young RA and Sharp PA: Let-7 represses Nr6al and a mid-gestation developmental program in adult fibroblasts. Genes Dev 27: 941-954, 2013.

16. Jiao LR, Frampton AE, Jacob J, Pellegrino L, Krell J, Giamas G, Tsim N, Vlavianos P, Cohen P, Ahmad R, et al: MicroRNAs targeting oncogenes are down-regulated in pancreatic malignant transformation from benign tumors. PLoS One 7: e32068, 2012.

17. Volinia S, Galasso M, Sana ME, Wise TF, Palatini J, Huebner K and Croce CM: Breast cancer signatures for invasiveness and prognosis defined by deep sequencing of microRNA. Proc Natl Acad Sci USA 109: 3024-3029, 2012.

18. Chang CJ, Hsu CC, Chang CH, Tsai LL, Chang YC, Lu SW, $\mathrm{Yu} \mathrm{CH}$, Huang HS, Wang JJ, Tsai $\mathrm{CH}$, et al: Let-7d functions as novel regulator of epithelial-mesenchymal transition and chemoresistant property in oral cancer. Oncol Rep 26: 1003-1010, 2011.

19. Ramberg H, Alshbib A, Berge V, Svindland A and Taskén KA: Regulation of PBX3 expression by androgen and Let-7d in prostate cancer. Mol Cancer 10: 50, 2011.

20. Childs G, Fazzari M, Kung G, Kawachi N, Brandwein-Gensler M, McLemore M, Chen Q, Burk RD, Smith RV, Prystowsky MB, et al: Low-level expression of microRNAs let-7d and miR-205 are prognostic markers of head and neck squamous cell carcinoma. Am J Pathol 174: 736-745, 2009.

21. Tezcan G, Tunca B, Bekar A, Yalcin M, Sahin S, Budak F, Cecener G, Egeli U, Demir C, Guvenc G, et al: Ficus carica latex prevents invasion through induction of let-7d expression in GBM cell lines. Cell Mol Neurobiol 35: 175-187, 2015.

22. Nuovo GJ, Garofalo M, Valeri N, Roulstone V, Volinia S, Cohn DE, Phelps M, Harrington KJ, Vile R, Melcher A, et al: Reovirus-associated reduction of microRNA-let-7d is related to the increased apoptotic death of cancer cells in clinical samples. Mod Pathol 25: 1333-1344, 2012.

23. Ivo D'Urso P, Fernando D'Urso O, Damiano Gianfreda C, Mezzolla V, Storelli C and Marsigliante S: miR-15b and miR-21 as circulating biomarkers for diagnosis of glioma. Curr Genomics 16: 304-311, 2015.

24. Yu Y, Nangia-Makker P, Farhana L, G Rajendra S, Levi E and Majumdar AP: miR-21 and miR-145 cooperation in regulation of colon cancer stem cells. Mol Cancer 14: 98, 2015.

25. Huang W, Lin J and Zhang H: miR-126: A novel regulator in colon cancer. Biomed Rep 4: 131-134, 2016.

26. Madison BB, Jeganathan AN, Mizuno R, Winslow MM, Castells A, Cuatrecasas $M$ and Rustgi AK: Let-7 represses carcinogenesis and a stem cell phenotype in the intestine via regulation of Hmga2. PLoS Genet 11: e1005408, 2015. 
27. Karin M and Greten FR: NNF-kappaB: Linking inflammation and immunity to cancer development and progression. Nat Rev Immunol 5: 749-759, 2005.

28. Lah TT, Babnik J, Schiffmann E, Turk V and Skaleric U: Cysteine proteinases and inhibitors in inflammation: Their role in periodontal disease. J Periodontol 64 (Suppl): 485-491, 1993.

29. Travis J and Potempa J: Bacterial proteinases as targets for the development of second-generation antibiotics. Biochim Biophys Acta 1477: 35-50, 2000.

30. Koblinski JE, Ahram M and Sloane BF: Unraveling the role of proteases in cancer. Clin Chim Acta 291: 113-135, 2000.

31. Barrett AJ: The cystatins: A diverse superfamily of cysteine peptidase inhibitors. Biomed Biochim Acta 45: 1363-1374, 1986.
32. Lindahl P, Abrahamson $\mathrm{M}$ and Björk I: Interaction of recombinant human cystatin $\mathrm{C}$ with the cysteine proteinases papain and actinidin. Biochem J 281: 49-55, 1992.

33. Dickinson DP, Thiesse M, Dempsey LD and Millar SJ: Genomic cloning, physical mapping, and expression of human type 2 cystatin genes. Crit Rev Oral Biol Med 4: 573-580, 1993.

34. Kim JT, Lee SJ, Kang MA, Park JE, Kim BY, Yoon DY, Yang Y, Lee CH, Yeom YI, Choe YK, et al: Cystatin SN neutralizes the inhibitory effect of cystatin C on cathepsin B activity. Cell Death Dis 4: e974, 2013. 